\title{
Halos around Herbig Ae/Be stars - more common than for the less massive $T$ Tauri stars
}

\author{
Ch. Leinert ${ }^{1}$, M. Haas ${ }^{1}$, P. Ábrahám ${ }^{1,3}$, and A. Richichi ${ }^{2, \star}$ \\ 1 Max-Planck-Institut für Astronomie, Königstuhl 17, 69117 Heidelberg, Germany \\ 2 European Southern Observatory, Karl-Schwarzschild-Strasse 2, 85748 Garching, Germany \\ 3 Konkoly Observatory of the Hungarian Academy of Sciences, PO Box 67, 1525 Budapest, Hungary \\ Received 5 March 2001 / Accepted 12 June 2001
}

\begin{abstract}
We present sub-arcsec observations of a sample of 31 Herbig Ae/Be and related stars obtained with the technique of near-infrared speckle interferometry. In 18 of the 31 stars we find evidence for circumstellar halos. These halos have a typical size of $1.1^{\prime \prime}$, corresponding to $\approx 1000 \mathrm{AU}$ at the distances of the objects. Most of the halos have blue colours, hence are seen in scattered light. The incidence of observable circumstellar matter is significantly higher than in a larger sample of $\mathrm{T}$ Tauri stars observed with the same technique (Leinert et al. 1993a). We suggest that this difference results as a consequence of a faster initial evolution of the more massive Herbig Ae/Be stars. The near-infrared halos found in our study appear to correspond to the "envelopes" invoked to model the strong mid-infrared excesses of Herbig Ae/Be stars.
\end{abstract}

Key words. stars: circumstellar matter - stars: pre-main-sequence

\section{Introduction}

Herbig Ae/Be stars are the more massive counterparts of T Tauri stars, and they show similar signs of stellar activity. It may be expected that the formation processes for these two groups of stars are similar as well.

For the young, low-mass T Tauri stars, observations have shown that they are surrounded by circumstellar disks, formed during the collapse of the protostellar cloud. This disk provides an important reservoir for the star, from which it draws a large fraction of its mass. In the initial phases of accretion, the disk dominates the luminosity of the system. Later, the disk becomes "passive" and is heated by radiation from the newly-formed young star, which still leads to substantial excess emission at infrared wavelengths. The infrared spectral energy distributions (SEDs) indicate that in many T Tauri stars the disk temperature decreases quite slowly with distance from the star. A simple explanation for these flat temperature profiles has been given by Natta (1995): thin, nebulous halos surrounding star and disk scatter the stellar radiation and thus provide additional heating of the outer parts of the disk.

Herbig Ae/Be stars by their original definition (Herbig 1960) are associated with mass concentrations giving rise to reflection nebulae, and in addition they often are

Send offprint requests to: Ch. Leinert,

e-mail: leinert@mpia-hd.mpg.de

* Based on observations obtained at the Calar Alto $3.5 \mathrm{~m}$ and the ESO $3.6 \mathrm{~m}$ telescopes. surrounded by substantial amounts of circumstellar matter. This is part of their general similarity to T Tauri stars. However, it has been questioned whether the disk-based scenario accepted for the formation of low-mass stars also should apply to the Herbig Ae/Be stars, as pointedly formulated by Hartmann et al. (1993) in the title of their paper: "The excess infrared emission of Herbig Ae/Be stars Disks or envelopes?". This should not be taken as a fully exclusive alternative, but as an encouragement to study the relative importance of envelopes and disks for star formation in the mass regimes of Herbig Ae/Be stars and of T Tauri stars. High spatial resolution observations are the most direct way to access this question.

Our first observations showed strong near-infrared halos around a few Herbig Ae/Be stars (LkH $\alpha$ 198, V376 Cas and AFGL 490, see Leinert et al. 1991a; Haas et al. 1992). Here we will report on near-infrared speckle observations of a sample of Herbig Ae/Be stars which we searched systematically for extended emission on the scale of a few hundred AU. The aim is to see if there are differences in the circumstellar matter distribution between Herbig Ae/Be stars and the lower mass $\mathrm{T}$ Tauri stars studied earlier, and if so, to understand the reason for this difference.

\section{Sample and data}

The sample of 31 Herbig Ae/Be and related stars studied with near-infrared speckle-interferometry was based on the list of Finkenzeller \& Mundt (1984), with emphasis 
on selecting apparently active objects. It is the same sample that was analysed by us for duplicity in a previous paper (Leinert et al. 1997, Paper I). In fact, we use here the same data to search for the incidence of halos.

The observations were performed with the $3.5 \mathrm{~m}$ telescope on Calar Alto and with the $3.6 \mathrm{~m}$ telescope on La Silla between 1985 and 1995. The sample, the journal of observations and the general method of near-infrared speckle-interferometry have been described in Paper I. A preliminary report on resolved emission found around some of these stars was given at the Amsterdam 1993 conference on Herbig Ae/Be stars (Leinert et al. 1994).

For speckle-interferometric data, the spatial information is best derived from the "visibility", i.e. the modulus of the Fourier transform of the object brightness distribution. The basic signature of extended emission is a drop of the visibility at low spatial frequencies (e.g. 1-2 $\operatorname{arcsec}^{-1}$ ), followed at higher frequencies by a constant plateau which represents the unresolved contribution from the star and its closest vicinity (see Fig. 1). For quantitative evaluation of the data, we approximate the brightness distribution of the halos by a Gaussian, normalised to the total object brightness:

$I(x)=\frac{I_{\text {halo }}}{I_{\text {tot }}} \cdot \frac{1}{\sqrt{2 \pi} \sigma} \exp \left(-\frac{x^{2}}{2 \sigma^{2}}\right)$,

where $\mathrm{x}$ and $\sigma$ are measured in arcsec, and the full width at half maximum brightness is $F W H M=2.35 \sigma$. For onedimensional slit scans, the method mostly used in our observations, this usually is an adequate representation also for more structured two-dimensional brightness distributions (Ridgway et al. 1986). The visibility for this Gaussian brightness distribution, when normalised to the total brightness $I_{\text {tot }}$, results as

$\tilde{I}(u)=\frac{I_{\text {halo }}}{I_{\text {tot }}} \cdot \exp \left(-2 \pi^{2} \sigma^{2} u^{2}\right)$

where $\mathrm{u}$ is the spatial frequency in $\operatorname{arcsec}^{-1}$. If such a halo symmetrically surrounds an unresolved source, the visibilities of the two components simply add, and a system visibility like those shown in Fig. 1 results. A halo around a binary would affect the system visibility curve in a corresponding, similar way (see Figs. 2 and 3 in Leinert \& Haas 1989).

Our standard procedure for the study of halos in this sample consisted of assuming a Gaussian halo brightness distribution centered on an unresolved source (or on a binary composed of unresolved sources) and solving by a least squares fit for the observables $I_{\text {halo }} / I_{\text {tot }}$ and $\sigma$. In a few cases, two Gaussian distributions of different $F W H M$, a narrow one and an extended one, were needed to appropriately fit the data. In this case, the total halo brightness is the sum of the brightnesses of the two halo components. Again, if both of these halos symmetrically surround an unresolved source, the visibilities of the three components simply add. Errors in halo parameters were determined from separate fits to subsets of the data (Dyck et al. 1984).

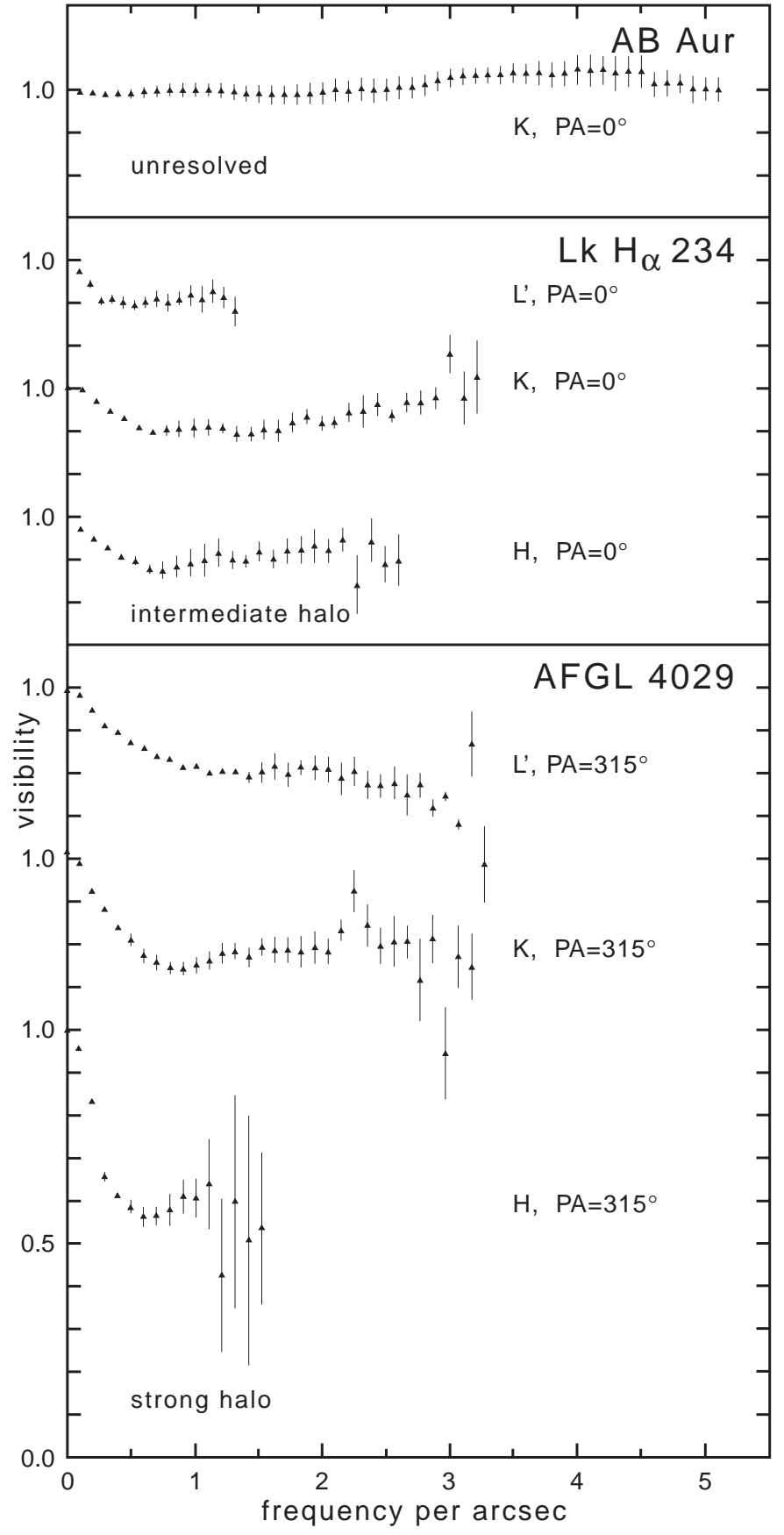

Fig. 1. Examples of one-dimensional visibility curves. They refer to an unresolved source (AB Aur, $K=4.3 \mathrm{mag}$ ), a source with an intermediate halo with little wavelength dependence (Lk H $\alpha 234, K=8.3 \mathrm{mag}$ ) and a source with a strong "blue" halo (AFGL 4029, $K=7.8 \mathrm{mag}$ ). In all cases, the visibility scale is 0.1 per tick mark.

Measurements at $2.2 \mu \mathrm{m}$ ( $K$ band) are available for all of the sources. For several objects, the search for halo emission included two or more of the near-infrared wavelength bands between $J$ and $M$, which in these cases allowed to study the wavelength dependence of the halo emission.

The objects cover the $K$ magnitude range from $3.5 \mathrm{mag}$ to $9.5 \mathrm{mag}$. Observations of good quality, with visibilities measured to spatial frequencies of $3 \operatorname{arcsec}^{-1}$ or more, 
Table 1. Summary of the survey for halos: (1) object, (2) distance and (3) spectral type (see Paper I), (4) fractional halo brightness in the band indicated in Col. (9), (5) to (8) geometry of the halo, (9) wavelength bands observed; data refer to the one shown in boldface, (10) wavelength dependence of fractional halo brightness, (11) scattering mass (dust plus gas), calculated according to Leinert et al. (1991a), (12) characterisation of the halo or the source, (13) references. For two-component models of the halo, these two components are referred to separately in Cols. (4) to (6) and occasionally also in (7) and (8). The remaining columns always refer to the full halo or system. Uncertain results are qualified by ":" (see text).

\begin{tabular}{|c|c|c|c|c|c|c|c|c|c|c|c|c|}
\hline Name & $\begin{array}{l}d \\
(\mathrm{kpc})\end{array}$ & $\begin{array}{r}\text { Spectral } \\
\text { type }\end{array}$ & $I_{\text {halo }} / I_{\text {tot }}$ & $\begin{array}{l}\text { Halo: } F v \\
\left({ }^{\prime \prime}\right)\end{array}$ & $\begin{array}{l}H M \\
(\mathrm{AU})\end{array}$ & $\begin{array}{l}\text { Elon- } \\
\text { gation }\end{array}$ & $\begin{array}{c}\text { Position } \\
\text { angle }\end{array}$ & $\begin{array}{c}\text { Observed } \\
\text { bands }\end{array}$ & $\begin{array}{c}\text { Relative } \\
\text { spectrum }\end{array}$ & $\begin{array}{c}\text { Scattering } \\
\text { mass }\left(M_{\odot}\right)\end{array}$ & $\begin{array}{l}\text { Character of } \\
\text { halo or source }\end{array}$ & $\begin{array}{l}\text { Refer- } \\
\text { ences }\end{array}$ \\
\hline$(1)$ & $(2)$ & $(3)$ & $(4)$ & $(5)$ & $(6)$ & $(7)$ & $(8)$ & $(9)$ & $(10)$ & $(11)$ & $(12)$ & $(13)$ \\
\hline $\mathrm{LkH} \alpha 198$ & 0.6 & $\mathrm{~B} / \mathrm{Ae}$ & $\begin{array}{l}0.19 \pm 0.03 \\
0.23 \pm 0.02\end{array}$ & $\begin{array}{l}0.50 \pm 0.07 \\
1.65 \pm 0.18\end{array}$ & $\begin{array}{l}300 \\
990\end{array}$ & no & & $J \boldsymbol{H} K L^{\prime}$ & $\lambda^{-2.0}$ & $5.6 \times 10^{-3}$ & strong & 1 \\
\hline V376 Cas & 0.6 & $\mathrm{~B} 5 \mathrm{e}$ & $\begin{array}{l}0.13 \pm 0.03 \\
0.18 \pm 0.02\end{array}$ & $\begin{array}{l}0.49 \pm 0.09 \\
2.28 \pm 0.10\end{array}$ & $\begin{array}{r}290 \\
1370\end{array}$ & $\begin{array}{c}\text { no } \\
\text { slight }\end{array}$ & $220^{\circ}$ & $\boldsymbol{H} K L^{\prime}$ & $\lambda^{-2.5}$ & $8.6 \times 10^{-3}$ & strong & 1 \\
\hline $\begin{array}{l}\mathrm{BD}+61^{\circ} 154 \\
\left.\mathrm{AFGL} 4029^{a}\right)\end{array}$ & $\begin{array}{l}0.65 \\
2.2\end{array}$ & $\begin{array}{r}\text { B:e } \\
\text { Be }\end{array}$ & $\begin{array}{l}0.12 \pm 0.03: \\
0.22 \pm 0.03\end{array}$ & $\begin{array}{c}0.7 \pm 0.2: \\
1.4 \pm 0.3\end{array}$ & $\begin{array}{r}450: \\
3100\end{array}$ & $\begin{array}{c}\text { uncertain } \\
\text { no }\end{array}$ & $\begin{array}{l}\text { E-W: } \\
315^{\circ}\end{array}$ & $\begin{array}{l}\boldsymbol{H} K L^{\prime} \\
H \boldsymbol{K} L^{\prime}\end{array}$ & $\lambda^{-1.0}$ & $\begin{array}{c}2.4 \times 10^{-3}: \\
0.50\end{array}$ & $\begin{array}{c}\text { intermediate } \\
\text { strong }\end{array}$ & \\
\hline AFGL $490^{a)}$ & 0.9 & $\mathrm{~B} 4$ & $0.30 \pm 0.03$ & $1.16 \pm 0.11$ & 1040 & no & $225^{\circ}$ & $\boldsymbol{H} K L^{\prime} M$ & $\lambda^{-1.2}$ & 0.045 & strong & 2 \\
\hline Elias 1 & 0.14 & $\mathrm{~A} 6 \mathrm{e}$ & $0.11 \pm 0.02$ & $1.1 \pm 0.2$ & 150 & yes & E-W & $\mathrm{J} \boldsymbol{H} K L^{\prime}$ & $\lambda^{-2.2}$ & $0.3 \times 10^{-3}$ & intermediate & 3 \\
\hline $\mathrm{LkH} \alpha 101$ & 0.8 & $\mathrm{~F} ? \mathrm{e}$ & $0.15 \pm 0.02$ & $0.9 \pm 0.2$ & 720 & & & $\boldsymbol{H} K L^{\prime} M$ & & & intermediate & \\
\hline AB Aur & 0.16 & $\mathrm{~B} 9, \mathrm{~A} 0 \mathrm{e}$ & $<0.02$ & & & & & $K$ & & $<0.1 \times 10^{-3}$ & unresolved & \\
\hline HK Ori & 0.46 & B8/A4ep & $<0.05$ & & & & & $J H \boldsymbol{K}$ & & $<2.1 \times 10^{-3}$ & binary & 4 \\
\hline $\mathrm{T}$ Ori & 0.46 & $\mathrm{~A} 3 \mathrm{e}$ & $<0.03$ & & & & & $K$ & & $<1.3 \times 10^{-3}$ & unresolved & 4 \\
\hline V 380 Ori & 0.46 & $\mathrm{~B} 8 / \mathrm{A} 1 \mathrm{e}$ & $<0.14$ & & & & & $\mathrm{~J} \boldsymbol{H} K L^{\prime}$ & & $<2.4 \times 10^{-3}$ & binary & 4 \\
\hline LkH $\alpha 208$ & 1.0 & $\mathrm{~F} 0 \mathrm{eV}$ & $<0.05$ & & & & & $K$ & & $<0.01$ & binary & 4 \\
\hline R Mon & 0.8 & $\mathrm{~B} 0 \mathrm{e}$ & $\begin{array}{l}0.21 \pm 0.02 \\
0.16 \pm 0.02\end{array}$ & $\begin{array}{l}0.4 \pm 0.1 \\
3.0 \pm 0.3\end{array}$ & $\begin{array}{r}320 \\
2400\end{array}$ & slight & $\mathrm{N}$ & $\boldsymbol{H} K L^{\prime}$ & $\lambda^{-2.3}$ & 0.12 & strong & \\
\hline $\mathrm{LkH} \alpha 25$ & 0.8 & B8pe & $<0.10$ & & & & & $K$ & & $<0.01$ & unresolved & \\
\hline Z CMa & 1.15 & FUOR & $<0.03$ & & & & & $\boldsymbol{H} K L^{\prime} \mathrm{M}$ & & $<2.1 \times 10^{-3}$ & binary & 5,6 \\
\hline $13395-6153^{b)}$ & $?$ & & $0.09 \pm 0.02:$ & $1.6 \pm 0.4:$ & & & & $\boldsymbol{K} L^{\prime}$ & & & faint & \\
\hline HR 5999 & 0.15 & $\mathrm{~A} 7 \mathrm{e}$ & $<0.03$ & & & & & $J H \boldsymbol{K}$ & & $<0.1 \times 10^{-3}$ & binary & 4 \\
\hline V921 Sco & 0.16 & Bep? & $0.06 \pm 0.04:$ & $0.8 \pm 0.4:$ & 130 & & & $\boldsymbol{K} L^{\prime}$ & & $0.2 \times 10^{-3}$ & faint & 4 \\
\hline KK Oph & 0.16 & $\mathrm{~B}, \mathrm{Ae}$ & $<0.05$ & & & & & $H \boldsymbol{K} L^{\prime}$ & & $<0.2 \times 10^{-3}$ & binary & 4 \\
\hline $17216-3801^{b)}$ & $?$ & & $0.10 \pm 0.04:$ & $0.3 \pm 0.1:$ & & & & $K \boldsymbol{L}^{\prime}$ & & no scattering & intermediate & \\
\hline MWC 300 & 15.5 & $\mathrm{eq} / \mathrm{BIe}$ & $0.07 \pm 0.02$ & $1.1 \pm 0.3$ & 1700 & no & & $\boldsymbol{H} K L^{\prime}$ & & 0.02 & faint & \\
\hline VV Ser & 0.44 & $\mathrm{~B}, \mathrm{Ae}$ & $0.08 \pm 0.03$ & $0.6 \pm 0.2$ & 260 & slight & E-W & $J \boldsymbol{H} K$ & & $0.4 \times 10^{-3}$ & faint & \\
\hline $\mathrm{R} \mathrm{CrA}$ & 0.13 & A5:e & $0.08 \pm 0.02$ & $1.0 \pm 0.4$ & 130 & & & $H \boldsymbol{K} L$ & & $0.9 \times 10^{-3}$ & faint & \\
\hline T CrA & 0.13 & F0:e & $<0.08$ & & & & & $\boldsymbol{H} K L$ & & $<0.9 \times 10^{-3}$ & unresolved & \\
\hline $\mathrm{G} 70.7+1.2^{c)}$ & $1-4.5$ & B: & $0.21 \pm 0.02$ & $1.2 \pm 0.2$ & 2500 & & & $J H K \boldsymbol{L}^{\prime} M$ & $\lambda^{0.8}$ & no scattering & strong & \\
\hline $\mathrm{BD}+40^{\circ} 4124$ & 1.0 & $\mathrm{~B} 2,3 \mathrm{e}$ & $0.12 \pm 0.03:$ & $1.1 \pm 0.3:$ & 1100: & uncertain & N-S: & $\boldsymbol{H} K L^{\prime}$ & & 0.02 & intermediate & \\
\hline PV Cep & 0.6 & $\mathrm{~A} 5 \mathrm{e}$ & $<0.05$ & & & & & $K$ & & $<4 \times 10^{-3}$ & unresolved & \\
\hline V645 Cyg & $3.5-5.6$ & $\mathrm{O} 7 \mathrm{e}$ & $<0.03$ & & & & & $H \boldsymbol{K}$ & & $<0.12$ & unresolved & \\
\hline $\mathrm{LkH} \alpha 234$ & 1.0 & $\mathrm{~B} 5 / 7 \mathrm{e}$ & $0.10 \pm 0.02$ & $1.8 \pm 0.5$ & 1800 & yes & $\mathrm{N}-\mathrm{S}$ & $\boldsymbol{H} K L^{\prime}$ & $\lambda^{0}$ & 0.03 & intermediate & \\
\hline $\mathrm{LkH} \alpha 233$ & 0.88 & $\mathrm{~A} 7 \mathrm{e}$ & $\begin{array}{l}0.33 \pm 0.06 \\
0.17 \pm 0.02\end{array}$ & $\begin{array}{c}0.51 \pm 0.03 \\
2.2 \pm 0.3\end{array}$ & $\begin{array}{r}450 \\
1900\end{array}$ & $\begin{array}{l}\text { uncertain } \\
\text { uncertain }\end{array}$ & $\begin{array}{l}\text { NNE: } \\
\text { NNW: }\end{array}$ & $\boldsymbol{H} K L^{\prime}$ & $\lambda^{-3}$ & 0.01 & strong & 7 \\
\hline MWC 1080 & $1.1-2.2$ & $\mathrm{~B} 0 ? \mathrm{e}$ & $<0.02$ & & & & & $J H \boldsymbol{K} L^{\prime} M$ & & $<0.7 \times 10^{-3}$ & binary & 4 \\
\hline
\end{tabular}

${ }^{a}$ High mass ${ }^{b}$ IRAS high mass YSO candidate ${ }^{c}$ high mass YSO.

References: [1] Leinert et al. (1991a), [2] Haas et al. (1992), [3] Haas et al. (1997), [4] Leinert et al. (1997), [5] Koresko et al. (1991), [6] Haas et al. (1992),

[7] Leinert et al. (1997). 
were obtained down to a brightness of $K=8.5 \mathrm{mag}$ (see Fig. 1). In these cases halos more extended than by $0.2^{\prime \prime}$ FWHM are well characterised by the measurements. For the two fainter sources, $\mathrm{Lk} \mathrm{H} \alpha 208$ and $\mathrm{Lk} \mathrm{H} \alpha 25$, the measurements suffered from noise.

\section{Results}

\subsection{The full sample}

The results of our halo search are summarised in Table 1. The most striking detections have already been published separately: $\mathrm{LkH} \alpha 198$ and V376 Cas in Leinert et al. (1991a), AFGL 490 in Haas et al. (1992), LkH $\alpha 233$ in Leinert et al. (1993b), Elias 1 in Haas et al. (1997). The near-infrared halo around $\mathrm{R}$ Mon was first discussed by Beckwith et al. (1984). In our full sample of 31 Herbig $\mathrm{Ae} / \mathrm{Be}$ and related stars we have 18 objects where at least a faint extended brightness on arcsec scale is indicated. In general, these halos are quite symmetric. The typical (median) halo size is $F W H M=1.1^{\prime \prime}$, or, with an assumed average distance of $1 \mathrm{kpc}, F W H M \approx 1000 \mathrm{AU}$. No evidence for halos was found in the thirteen remaining sources within the limits given in Table 1. Fields for which no significant values can be derived from the data are left blank.

When measurements in several wavelength bands were available we have chosen to present results for the wavelength band with the best $\mathrm{S} / \mathrm{N}$ ratio. The wavelength dependence of halo brightness relative to the unresolved central source usually was derived from all observed wavelength bands. The exceptions are Elias 1, where at $K$ and $L^{\prime}$ we see a narrower structure possibly due to a close companion; G70.7+1.2, where the $J$ band, and LkH $\alpha 233$, where the $L^{\prime}$ band do not definitely show resolved emission.

A special source of uncertainties in the halo parameters are seeing variations during the measurements. Such variations to some extent can mimick the decay in visibility curves at low spatial frequencies typical for resolved halo emission. This in some cases leads to an unknown additional systematic error on the measurements. Where a significant systematic contribution of this error cannot be excluded, this has been indicated by the ":" sign following the halo parameters in Table 1 . In the case of V380 Ori, a $0.15^{\prime \prime}$ binary, the visibility curve in the $H$ band showed a small dip at low spatial frequencies superimposed on the cosine shape typical for a binary. In principle, this could be the signature of halo emission around this star. However, since from observations in the other wavelength bands we have no additional evidence supporting this assumption, we only give an upper limit for a possible halo contribution. The linear size of the halos is given on the basis of available distance determinations (see Paper I), and therefore is affected by the uncertainty of those determinations. The errors on the relative spectrum of halo brightness, expressed as error in the exponent of the adopted power law, are estimated as $\pm 0.3-0.4$. This error is comparatively large but sufficient to distinguish between halos dominated by scattering and those probably containing a substantial contribution of thermal emission.

Table 1 shows separate columns for elongation and position angle. The information under "elongation" refers to the question of whether we see an intrinsic flattening of the extended brightness distribution or whether we see none. In the case of a flattened distribution, such as for Elias 1 and $\mathrm{LkH} \alpha$ 234, the column "position angle" shows the orientation of the long axis. Even for quite circularly symmetric distributions of brightness, however, the center of the light distribution may be offset from the unresolved central source. In this case, denoted with elongation "no", the direction of this offset is given in the column "position angle", too.

\subsection{Comments on individual sources}

\subsection{1. $\mathrm{LkH} \alpha 198$ and $\mathrm{V} 376$ Cas}

In these sources, an additional argument for scattering as the main process of halo radiation comes from the strong polarisation of the halos observed in the near-infrared (Leinert et al. 1991a). For a more detailed overview on the complex geometry of these two neighbouring objects see the discussion in Leinert et al. (1991a) and the more recent studies by Koresko et al. (1997) and Asselin et al. (1996).

\subsubsection{AFGL 4029}

AFGL 4029 is surrounded by a strong halo (see Fig. 1): at all three wavebands the visibility declines at low frequencies followed by a nearly constant plateau. The height of the plateau is different for the three wavebands; it is smallest at $H$, meaning that the relative contribution of the halo increases at the shorter wavelengths. Also, at $H$ the decline is steepest, meaning that the halo extent is largest. These are typical signatures for a scattering halo.

Interestingly, for AFGL 4029, a source with a strong halo, the phase of the Fourier transform (not shown here), which contains the information about the halo symmetry, deviates from zero. This means that the halo is not located symmetrically around the central unresolved source, but is shifted towards $\mathrm{PA}=315^{\circ}$. This picture appears to be quite consistent with the $L^{\prime}$ and $K$ band data. At $H$, however, and to a lesser extent at $K$, a thorough inspection of the visibility reveals a faint dip at spatial frequency $0.6 \operatorname{arcsec}^{-1}$ and an - admittedly noisy - maximum at about $1.2 \operatorname{arcsec}^{-1}$. Together with the strong asymmetry in the phase, this suggests that we see two maxima in brightness. The possibility of a double star, embedded within a strong halo, can be excluded on the basis of the $K$ and $L^{\prime}$ band data. Rather AFGL 4029 could have an underlying bipolar structure. The unresolved central object dominates the flux and the extended emission is strongest in the NW direction, with a brightness minimum between its peak and the central source. Such a brightness 
distribution would naturally result with an optically thick circumstellar disk, which is responsible for a strongly asymmetrical extinction of an intrinsically bipolar halo, dimming one of the lobes (the southeastern in this case). Forward scattering also would tend to boost the brightness of the nearer lobe. This structure of the source has also been proposed by Deharveng et al. (1997) from an optical and near-infrared study of the region. The presence of both high optical thickness regions and low optical thickness regions around AFGL 4029 is also concluded from the mid-infrared observations of Zavagno et al. (1999).

\subsection{3. $\mathrm{LkH} \alpha 101$}

Recently, Tuthill et al. (2001) resolved $\mathrm{LkH} \alpha 101$ at $1.65 \mu \mathrm{m}$ and $2.2 \mu \mathrm{m}$ by aperture masking techniques on the Keck I telescope. They found a limb-brightened structure with a radius of 21 milli-arcsec (mas) for the circular ridge of brightest emission and in addition a probable companion at 180 mas to E-NE, contributing $4 \%$ to the system brightness at $\mathrm{H}$. They interpret the ridge as the location of the hot edge of an inner cavity in an accretion disk seen more or less face-on. The results, published as high-resolution maps over a field of $0.3^{\prime \prime} \times 0.3^{\prime \prime}$, show no evidence for the arcsec-sized halo found in our observations. Possibly their method is not sensitive to this wide structure. Our speckle observations, performed on a $3.5 \mathrm{~m}$ telescope, could not have resolved the limb-brightened structure and would not have shown the close, faint companion.

\subsubsection{AB Aur}

AB Aur is unresolved in our measurements: the visibility shown in Fig. 1 remains nearly constant, corresponding to a size of less than 30 mas. Millan-Gabet et al. (1999) were able to resolve this source in the near-infrared at the IOTA interferometer and found a Gaussian FWHM of $4.7 \pm 0.1$ mas. Marsh et al. (1995) infer a considerably larger extension of $\approx 0.3^{\prime \prime}$ at mid-infrared wavelengths between $10 \mu \mathrm{m}$ and $20 \mu \mathrm{m}$. In continuum mminterferometry AB Aur was unresolved, but measurements in the ${ }^{13} \mathrm{CO}(1 \rightarrow 0)$ line gave evidence for an inclined disk of $3^{\prime \prime}$ size (Mannings \& Sargent 1997). Ground-based (Nakajima \& Golimowski 1995) and HST coronographic observations (Grady et al. 1999) show extended nebulosity with the size of this $\mathrm{CO}$ disk; but in contrast to the mm observations, the HST images show a largely circularly - symmetric structure. Thus AB Aur gives a good example of how different observing methods are sensitive to different parts of the circumstellar matter distribution. It appears that on scales of $100 \mathrm{AU}$ (or roughly $1^{\prime \prime}$ ) there is insufficient dust around to appear as a scattering halo. However, the density distribution must be quite peaked towards the star in order to appear - probably in thermal emission - at the interferometric resolutions.

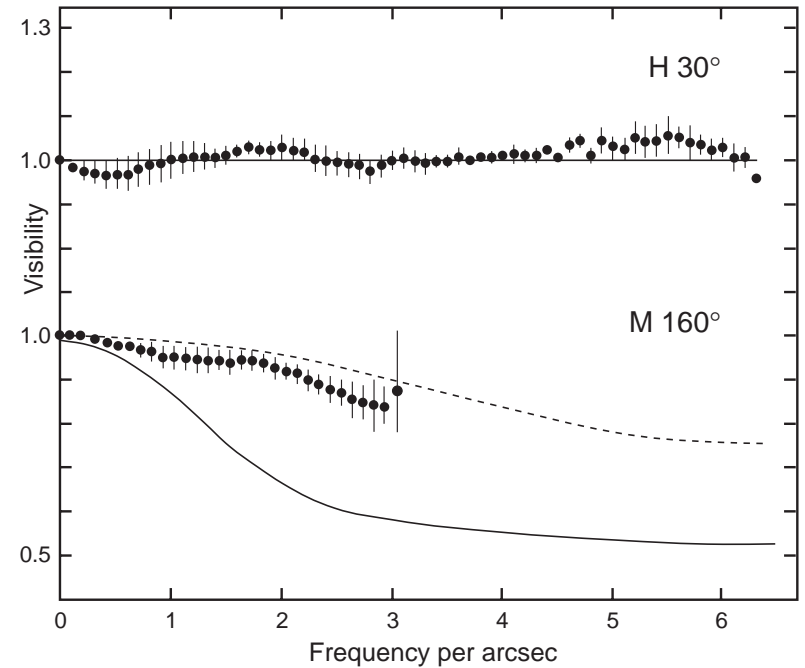

Fig. 2. Near-infrared speckle observations of Z CMa from Calar Alto. Top: the $H$ band $(1.65 \mu \mathrm{m})$ observations from February 13, 1992, performed at PA $30^{\circ}$, i.e. perpendicular to the line connecting the two components of $\mathrm{Z} \mathrm{CMa,} \mathrm{show}$ the source spatially unresolved in this direction. The line at visibility 1.0 corresponds to a perfect point source. Bottom: the $M$ band $(4.8 \mu \mathrm{m})$ visibility observations $(\bullet)$ at $\mathrm{PA} 160^{\circ}$ from January 7, 1993, are compared to the expectations for a binary with brightness ratio 0.15 (Koresko et al. 1991, dashed line) and a model including an extended disk-like structure in this system, as proposed by Malbet et al. (1993, solid line). Our observations support the binary model, but require a brightness ratio of 0.25 at the time of observations.

\subsubsection{R Mon}

This is a highly structured region with $\mathrm{R}$ Mon at the apex of the visible lobe of the underlying bipolar geometry. From HST WFPC2 and near-infrared adaptive optics observations, Close et al. (1997) detected a T Tauri companion star at $0.69^{\prime \prime} \pm 0.01^{\prime \prime}$, which is fainter by about $5 \mathrm{mag}$ in the near-infrared. Including previous determinations of the SED of the source, they also give a model of $\mathrm{R}$ Mon which, in addition to a close-in circumstellar disk, requires a denser envelope well within the orbit of the companion, and a thinner one extending well beyond. The model is not presented in a quantitative way. But the extent of these envelopes, the effective radii of which may be estimated from the qualitative information given in the paper to $\approx 0.5^{\prime \prime}$ and $\approx 2.5^{\prime \prime}$, appears to match the sizes of $0.4^{\prime \prime}$ and $3^{\prime \prime}$ given for our two halo components in Table 1.

\subsubsection{Z CMa}

$\mathrm{Z}$ CMa is a binary with a separation of $0.1^{\prime \prime}$ at $\mathrm{PA} 120^{\circ}$ (Koresko et al. 1991). These authors also found that the spectral energy distribution of $\mathrm{Z}$ CMa could best be modeled when including a circumbinary disk of size $\approx 400$ AU. Malbet et al. (1993) claimed discovery of an elongated emission region of this size with an orientation in $\mathrm{PA} 160^{\circ}$ from their observations in the $L^{\prime}(3.87 \mu \mathrm{m})$ 
and $M(4.75 \mu \mathrm{m})$ bands. Figure 2 shows that in our nearinfrared observations at $H$ no sign of extended emission can be seen in the direction perpendicular to the line connecting the two components of Z CMa. Similarly, our $M$ band $1 \mathrm{D}$-speckle observations at PA $160^{\circ}$, performed at higher spatial resolution than those of Malbet et al., do not show the proposed extended emission. The slightly decreasing visibility curve is naturally explained by the existence of the companion (see Fig. 2), if the brightness ratio is 0.25 , a little larger than the ratios of $0.14-0.22$ found previously (Koresko et al. 1991; Tessier et al. 1994). We conclude with the latter authors that our near-infrared study does not show any evidence for a resolved circumbinary disk-like structure around Z CMa.

\subsection{7. $\mathrm{Lk} \mathrm{H} \alpha 234$}

$\mathrm{LkH} \alpha 234$ lies in a complicated region with several young sources around (see, e.g. Weintraub et al. 1994; Fuente et al. 2001). The visibility curve shown in Fig. 1 declines at low frequencies from 1.0 to about 0.9 , and remains nearly constant at higher frequencies. This behaviour, signifying the presence of extended emission around an unresolved source, is similar in all three wavebands, with $F W H M=1.8^{\prime \prime} \pm 0.5^{\prime \prime}$. The decline is slightly steeper at $L^{\prime}$ than at $K$ and $H$. This means that the extent of the halo is larger at $L^{\prime}$ than at $K$ and $H$, which is not typical for a scattering halo, but which could result if heating of the circumstellar material plays a role in the emission. In our measurements, $\mathrm{Lk} \mathrm{H} \alpha 234$ is significantly less extended in east-west direction $\left(F W H M=0.6^{\prime \prime} \pm 0.4^{\prime \prime}\right)$. This suggests a flattened distribution extending northsouth. Indeed, the polarisation map of Weintraub et al. (1994) shows across $\mathrm{LkH} \alpha 234$ the parallel pattern expected for a bipolar geometry with a disk extending northsouth. In the case of $\mathrm{LkH} \alpha 234$, our data are consistent with a primarily disk-like structure of the circumstellar dust distribution.

\subsection{Relation to more recent studies}

Since our observing campaigns in 1995, a few high spatial resolution observations on Herbig Ae/Be stars have been performed with superior techniques.

Millan-Gabet et al. (2001) have studied 15 Herbig Ae/Be stars at near-infrared wavelengths with the Infrared Optical Telescope Array (IOTA) on Mount Hopkins with a baseline of up to $38 \mathrm{~m}$. They have four sources in common with our list: $\mathrm{AB}$ Aur, MWC 1080, T Ori and V 380 Ori. T Ori was found unresolved, as in our data. For AB Aur, V 380 Ori and MWC 1080 they found extended emission from regions several milliarcsec in size, which was not and could not have been resolved in our single dish data from a $3.5 \mathrm{~m}$ telescope. Although sensitive to smaller spatial extents than our survey, these interferometric data lead to a similar conclusion: the resolved near-infrared brightness distributions show no evidence for elongated bipolar or disk-like geometries.

The Hubble Space Telescope (HST) has observed only a few intermediate-mass young stars, with publications on R Mon (Close et al. 1997) and on AB Aur (Grady et al. 1999). Results relevant in our context have been mentioned in the section on individual sources above. The HST observations are sensitive to fainter nebulosity than our near-infrared study and therefore show both more complex detail and the structures of the regions lying farther out.

Corporon \& Lagrange (1999) report on the detection of six spectroscopic companions to Herbig Ae/Be stars, of which T Ori, MWC 300 and MWC 1080 appear in our list. In addition they have indirect evidence for spectroscopic companions from the identification of the Li6709 $\AA$ line in seven more Ae/Be stars, among which HK Ori and V 380 Ori belong to our sample. Because most of these companions probably are very close to the primary star, these new detections are important for the overall picture but have no obvious relation to the distribution of circumstellar material on the scales we are searching. However, we note that none of the five binaries common to our sample exhibits a strong halo.

Far-infrared studies from the Kuiper Airborne Observatory (Di Francesco et al. 1998) or from the ISO satellite (Ábrahám et al. 2000) have a spatial resolution of $60^{\prime \prime}-120^{\prime \prime}$, thereby covering regions which include much more distant material than our halos. In addition, in the crowded regions around many of our objects they may be contaminated by other sources. Therefore we do not consider the far-infrared emission further, although it may be very informative for a comprehensive picture of the objects. For similar reasons, we did not include in our discussion the extensive mm continuum mapping survey performed by Henning et al. (1998) on Herbig Ae/Be and FU Orionis stars with beam sizes of $11^{\prime \prime}-23^{\prime \prime}$. But we will come back to the recent findings from $\mathrm{mm}$ interferometry in Sect. 4.3.

\section{Discussion}

\subsection{Incidence of extended emission}

As already indicated in Table 1, we classify near-infrared halos as strong, if in one of the observed wavelength bands they contribute more than $20 \%$ to the system brightness, as faint, if this contribution is less than $10 \%$ but detected, and as intermediate in the intervening cases. With this classification we then find in our sample of 31 Herbig Ae/Be and related stars 7 objects with strong near-infrared halos, 6 with intermediate and 5 with faint halos; 7 sources are binary and 6 are unresolved. Thus more than a third of the sources have strong or intermediate halos and for more than half of them at least some extended emission on the arcsec scale can be detected.

This contrasts to the case of the low mass $\mathrm{T}$ Tauri stars, where we studied in Taurus a sample of 104 objects 
(Leinert et al. 1993a) with the same observing and reduction technique. Among these objects, only HL Tau shows a strong near-infrared halo (Beckwith et al. 1984), marginal detection of extended emission occured around DG Tau (see Leinert et al. 1991b) and extended emission of intermediate strength was found in the binary Haro 6-10 (Leinert \& Haas 1989). If we omit the 33 sources fainter than $K=8.5 \mathrm{mag}$ from the Taurus sample, in order to have a better matching of the brightness range with the Herbig Ae/Be sample, the percentage of $\mathrm{T}$ Tauri stars with halo still is only $3 / 71 \approx 5 \%$. This low frequency of observable near-infrared halos around the $\mathrm{T}$ Tauri stars in Taurus does not seem to be untypical: in the younger and more embedded population of T Tauri stars in the $\rho$ Ophiuchi cloud, among the brighter objects accessible to speckle interferometric observations, also only few extended sources are known from near-infrared observations: Elias 21, Elias 29, WL16 and YLW 16A (Zinnecker et al. 1988). This Ophiuchus sample also contains about 100 stars, leading to essentially the same conclusion regarding halo frequency around $\mathrm{T}$ Tauri stars like the Taurus sample.

We therefore conclude that halos occur significantly more frequently in the intermediate mass Herbig Ae/Be stars than in the low mass $\mathrm{T}$ Tauri stars. This is true in particular for the strong halos.

The larger luminosity of Herbig Ae/Be stars with respect to T Tauri stars should not per se influence the detectability of circumstellar extended emission, since the detection is in the relative flux coming from these halos. Primarily, the detection is therefore limited neither by spatial resolution nor by source brightness, but by contrast and stability of observing conditions. With our technique we should have found the halos contributing more than $5 \%$ to the system brightness. However, for sources fainter than $K=8.5 \mathrm{mag}$, the noise in the signal may hide all the signatures of faint and intermediate halos. This is why we limited the comparison between Herbig Ae/Be and $\mathrm{T}$ Tauri stars to magnitudes brighter than this limit.

Among the seven binaries in Table 1, we have no definitive detection of halo emission. In part this may be a selection effect, since faint and sometimes even intermediate halos are difficult to detect in binaries with separation similar to the halo size, although we found one in the T Tauri star Haro 6-10. However, there are also physical reasons why halos could be rare in binaries: the orbital motion of the binary components may lead to a faster dispersal of circumstellar matter, as was concluded from mm continuum observations of single and binary $\mathrm{T}$ Tauri stars (Osterloh \& Beckwith 1995). Given the similar incidence of companions in T Tauri and Herbig Ae/Be stars, no significant influence of duplicity on the comparison between the two groups is expected.

We suggest an explanation of the higher incidence of halos among Herbig Ae/Be stars based on different evolutionary time scales: the Herbig Ae/Be stars on the average develop faster and are still younger at the time we observe them. E.g., taking the evolutionary tracks of
D'Antona \& Mazzitelli (1994), a star of $2.5 M_{\odot}$ will start approaching the main sequence at an age of $10^{6}$ years and reach it at $5 \times 10^{6}$ years, while a young star of mass $1 M_{\odot}$ or less will spend a much longer time on its track towards the main sequence. The chances that the diffuse material from the parental cloud core, swirling around the young star, has not yet been fully accreted or has not yet been dispersed are therefore more favourable in the case of Herbig Ae/Be stars than for the more slowly evolving and longer-lived T Tauri stars. This could lead to the significantly higher frequency of circumstellar halos in the Ae/Be stars.

\subsection{Nature of the extended near-infrared emission: Morphology and relation to mid-infrared emission}

There is ample evidence for circumstellar disks in $\mathrm{T}$ Tauri stars, but our near-infrared speckle-interferometric surveys did not show evidence of the extended emission associated with them. The lack of disk-like extended emission in our sample of Herbig Ae/Be stars therefore does not preclude the existence of circumstellar disks in these objects. Such disks may play an important role also for the structure of these more massive systems (Waters \& Waelkens 1998; Natta et al. 2000), but our observations would not tell, because they are not sensitive to those particular spatial structures.

When we studied the extended emission around a source in more than one (scanning) direction, the derived sizes depended very little on position angle, which indicates a more or less circularly symmetric brightness distribution. This was also true for sources where images at visual wavelengths clearly show bipolar structure probably in combination with an edge-on or moderately inclined disk, like for $\mathrm{LkH} \alpha 233$ or V 376 Cas. The notable exception is Elias 1 (Haas et al. 1997) which at $J$ and $H$ shows a narrow structure elongated east-west. However, even in this case it is not clear whether we are seeing radiation from a circumstellar disk. $\mathrm{LkH} \alpha 234$ also shows a substantial elongation, in this case in north-south direction; the remaining elongations and preferential directions given in Table 1 for other sources are not very pronounced.

The roughly symmetrical shape of the resolved nearinfrared brightness in our objects suggests that the circumstellar material fills a large part of the circumstellar volume. Lobes in a bipolar geometry, confined by denser material with conical openings, highly flared extensions of disks, denser doughnut-shaped as well as thinner more isotropic distributions all would be natural contributors to this appearance. In the combined effect, the circumstellar matter we observe as halos must be optically thick at visual wavelengths, otherwise it could not contribute substantially to the near-infrared flux. Most of the sources nevertheless can be well studied in the visual, which speaks for an anisotropic distribution of the circumstellar material within the first few hundred $\mathrm{AU}$ from the star. The halos cannot continuously surround the star but must contain substantial regions of lower optical thickness. 

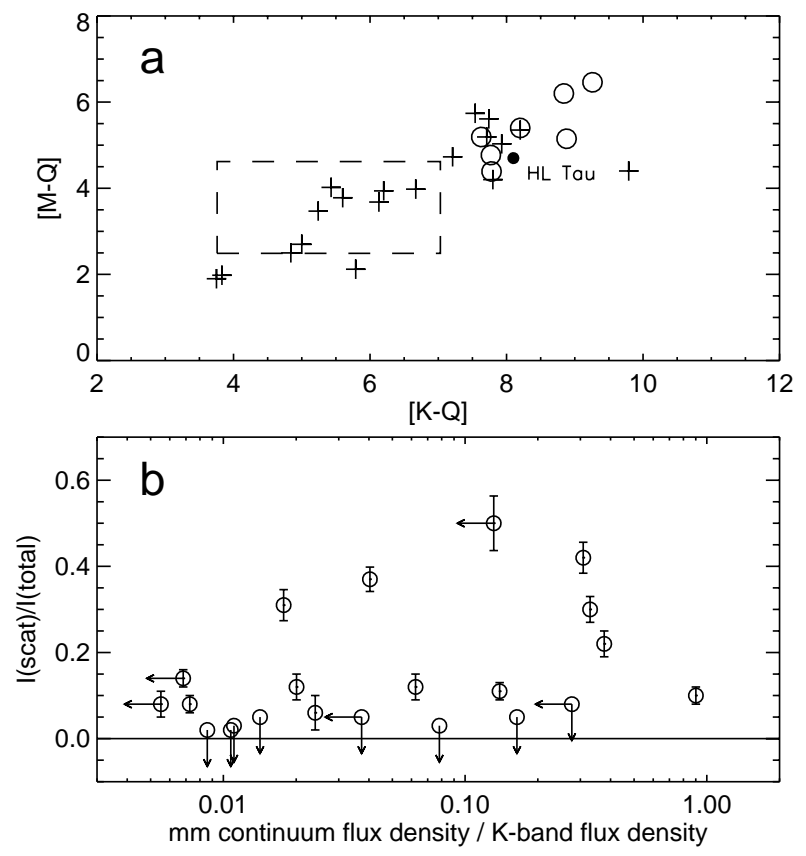

Fig. 3. a) mid-infrared color-color diagram of those sources from Table 1 where KMQ photometry was available in the literature. Symbols: Herbig Ae/Be stars with strong halo (o), with faint and intermediate halo $(+)$. For comparison: HL Tau $(\bullet)$ is an extreme T Tauri star, and the only one in Taurus with a strong halo. The dashed rectangle marks the area populated by normal T Tauri stars, including flat spectrum sources. b) correlation diagram between the relative intensity of the observed halos and the mm continuum $(1.1 \mathrm{~mm}$ or $1.3 \mathrm{~mm}$ flux density), normalised by the $K$ band flux (which is dominated by the stellar emission).

Further clues about the nature of the extended emission come from a comparison with published radiative transfer calculations. Most recent models predicting the spectral energy distribution of Herbig $\mathrm{Ae} / \mathrm{Be}$ stars build on both a compact dense circumstellar disk and an optically thin more extended envelope (e.g. Miroshnichenko et al. 1999). Note that the "envelopes" in these models refer to the same geometrical distribution as our "halos". The models predict that, due to their shallower temperature dependence with radial distance, the envelopes dominate the extended near-infrared and often the total mid-infrared brightness of the system, while the disks are conspicuous at far-infrared/submm $/ \mathrm{mm}$ wavelengths. The characteristic sizes of these model envelopes are in the order of 500-1000 AU, corresponding to $0.5^{\prime \prime}-1.0^{\prime \prime}$ at an average distance of $1 \mathrm{kpc}$, which is in excellent agreement with the sizes of the observed halos (see Table 1). Therefore it is tempting to identify the envelopes used by e.g. the models of Miroshnichenko et al. (1999) with the halos detected by us. The morphological information resulting from the near-infrared observations then provides empirical evidence for the size of these envelopes, which is not so well constrained by the models themselves.
The identification of mid-infrared emitting envelopes with the observed near-infrared halos suggests a correlation between strength of the halo and mid-infrared properties of Herbig Ae/Be stars. In Fig. 3a we plotted a mid-infrared colour-colour diagram of those sources from Table 1 where KMQ photometry was available in the literature. The figure reveals an interesting trend: sources with strong halos $\left(I_{\text {halo }} / I_{\text {tot }} \geq 0.2\right)$ occupy the reddest part of the diagram, beyond the locus of even the most active $\mathrm{T}$ Tauri stars like HL Tau. (Also in other colour-colour plots, like $\mathrm{K}-\mathrm{N} / \mathrm{K}-\mathrm{Q}$ or $\mathrm{M}-\mathrm{N} / \mathrm{N}-\mathrm{Q}$, the trend is clearly seen.)

According to the model calculations of Miroshnichenko et al. (1999, see their Fig. 1) a high mid-infrared spectral index indicates that the bulk of the mid-infrared emission is produced by an envelope, while for sources of lower spectral index the contributions from disk and envelope are comparable. The high mid-infrared emission also indicates that a significant amount of material is relatively far from the star, i.e. the envelopes observable in scattered light are not only strong, but also extended on the 100 AU scale, perhaps due to a relatively shallow radial density distribution. Sources with weak or non-detectable halos are therefore those having either a weak tenuous envelope, or having an envelope so strongly peaked that our techniques cannot resolve it. A further possibility for the suppression of extended mid-infrared emission was suggested by Meeus et al. (2001) from a discussion of the SEDs in a sample of isolated Herbig Ae/Be stars: if the circumstellar matter distribution contains a geometrically and optically thick structure close to the star, then the outer regions will be shadowed and neither enhanced mid-infrared excesses nor near-infrared halos will be observed.

Still, the question remains whether the observed strong near-infrared halos also require the presence of large amounts of dense circumstellar matter, of which they would simply be the optically thinner extension. To this end we plotted in Fig. 3b a correlation diagram between the relative intensity of the halos and the $\mathrm{mm}$ continuum flux density $(1.1 \mathrm{~mm}$ or $1.3 \mathrm{~mm})$ of the system. Detection of mm flux in these optically visible objects requires that the circumstellar dust be distributed in dense, highly flattened structures. The lack of any obvious correlation in Fig. 3b supports our conclusion that our observed halos are a phenomenon different from and at most weakly related to the circumstellar disks.

\subsection{Herbig Ae stars and Herbig Be stars}

Recently, Natta et al. (2000) have emphasised a structural difference between Herbig Ae/Be stars with spectral types later than B8 (the Herbig Ae stars) and those with earlier spectral types (the Herbig Be stars). While almost all of the Herbig Ae stars studied by them were detected in $\mathrm{mm}$ interferometric measurements, this was the case for only one out of seven Herbig Be stars. These authors suggest 
that in the latter group the strong stellar radiation field initiates a rapid evolution of the circumstellar environment, depleting a larger volume from dust concentrations in disks.

In our data, there is no such pronounced difference regarding the occurence of halos between these two subgroups of stars. Nevertheless there are indications that with respect to halos these two subgroups are not identical. For most of the early-type stars in our sample we detected extended near-infrared emission on the scale of several hundred $\mathrm{AU}$, with a median value of 0.1 for the fractional halo brightness. For most of the Herbig Ae stars, on the other hand, no halo could be detected, and the median result then is a non-detection.

This finding suggests again that these near-infrared halos have little structural relation with the typical circumstellar disks. The underlying reason for the indicated difference between Herbig Ae and Herbig Be stars may be the same as that which we propose to be responsible for the difference of intermediate mass stars to $\mathrm{T}$ Tauri stars: that the faster evolution of the more massive stars allows more remnant circumstellar material at the outer reaches of the close stellar environment to be present in recognisable amounts. It will be worthwhile studying how this apparent richness of circumstellar material around the most massive Herbig Ae/Be stars should be combined with the "inside" destructive action advocated by Natta et al. (2000).

\section{Conclusions}

From our near-infrared speckle-interferometric study of a sample of active Herbig Ae/Be stars we conclude:

1. Halos on an arcsec scale are much more common around young stars of intermediate mass than around $\mathrm{T}$ Tauri stars;

2. This finding could simply be a result of the faster evolution of the more massive Ae/Be stars;

3. Within our sample of Herbig Ae/Be stars it is the earlier spectral types which more often show detectable near-infrared halos;

4. We classify the observed extended emission as halos because in almost all cases it appears roughly symmetrical, with little dependence of size on position angle; Elias 1 and Lk H $\alpha 234$ are exceptions, but in both the extended emission is only of moderate strength;

5. Based on the observed wavelength dependence of the extended emission, most of these halos are seen in scattered light. Estimates for the amount of scattering material typically result in $10^{-3} M_{\odot}-10^{-2} M_{\odot}$;

6. The halos can be identified with the envelopes assumed in recent modellings of the spectral energy distributions of Herbig Ae/Be stars to explain the strong midinfrared excesses. Sources without observable nearinfrared halos have probably either too weak or too centrally peaked envelopes or have the halo shadowed by optically thick material lying closer to the central star;

7. No obvious relation between the occurence of halos and of circumstellar disks was found.

Acknowledgements. We want to thank the staff of the Calar Alto and La Silla observatories for good and enduring support during the various observing runs contributing to this paper. Ch. L. is particularly grateful to Uwe Graser who took over much of the daily work in another joint project and thus made available the time to finalise this study. We thank the referee, J. P. Berger, for many helpful comments and suggestions and Th. Henning for stimulating discussions. This work made use of the Simbad data base.

\section{References}

Ábrahám, P., Leinert, Ch., Burkert, A., Henning, Th., \& Lemke, D. 2000, A\&A, 354, 965

Asselin, L., Ménard, F., Bastien, P., Monin, J.-L., \& Rouan, D. 1996, ApJ, 472, 349

Beckwith, S. V. W., Zuckerman, B., Skrutskie, M. F., \& Dyck, H. M. 1984, ApJ, 287, 793

Close, L. M., Roddier, F., Hora, J. L., et al. 1997, ApJ, 489, 210

Corporon, P., \& Lagrange, A.-M. 1999, A\&AS, 136, 429

D’Antona, F., \& Mazzitelli, I. 1994, ApJS, 90, 467

Deharveng, L., Zavagno, A., Cruz-González, et al. 1997, A\&A, 317,459

Di Francesco, J., Evans II, N. J., Harvey, P. M., Mundy, L. G., \& Butner, H. M. 1998, ApJ, 509, 324

Dyck, H. M., Zuckerman, B., Leinert, Ch., \& Beckwith, S. 1984, ApJ, 287, 801

Finkenzeller, U., \& Mundt, R. 1984, A\&AS, 55, 109

Fuente, A., Neri, R., Martín-Pintado, J., et al. 2001, A\&A, in press

Grady, C. A., Woodgate, B., Bruhweiler, F. C., et al. 1999, ApJ, 523, L151

Hartmann, L. K., Kenyon, S. J., \& Calvet, N. 1993, ApJ, 407, 219

Haas, M., Leinert, Ch., \& Lenzen, R. 1992, A\&A, 261, 130

Haas, M., Christou, J. C., Zinnecker, H., Ridgway, S. T., \& Leinert, Ch. 1992, A\&A, 269, 290

Haas, M., Leinert, Ch., \& Richichi, A. 1997, A\&A, 326, 1076

Henning, Th., Burkert, A., Launhardt, R., Leinert, Ch., \& Stecklum, B. 1998, A\&A, 336, 565

Herbig, G. H. 1960, ApJS, 4, 337

Koresko, C. D., Beckwith, S. V. W., Ghez, A. M., Matthews, K., \& Neugebauer, G. 1991, AJ, 102, 2073

Koresko, C. D., Harvey, P. M., Christou, J. C., \& Fugate, R. Q. 1997, ApJ, 485, 213

Leinert, Ch., \& Haas, M. 1989, ApJ, 342, L39

Leinert, Ch., Haas, M., \& Lenzen, R. 1991a, A\&A, 246, 180

Leinert, Ch., Haas, M., Richichi, A., Zinnecker, H., \& Mundt, R. 1991b, A\&A, 250, 407

Leinert, Ch., Zinnecker, H., Weitzel, N., et al. 1993a, A\&A, 278,129

Leinert, Ch., Haas, M., \& Weitzel, N. 1993b, A\&A, 271, 535

Leinert, Ch., Richichi, A., Weitzel, N., \& Haas, M. 1994, in The nature and evolutionary status of Herbig Ae/Be stars, ed. P. S. Thé, M. R. Pérez, \& E. P. J. van den Heuvel, ASP Conf. Ser., 62, 155 
Leinert, Ch., Richichi, A., \& Haas, M. 1997, A\&A, 318, 472, Paper I

Malbet, F., Rigaut, F., Bertout, C., \& Léna, P. 1993, A\&A, 271, L9

Mannings, V., \& Sargent, A. I. 1997, ApJ, 490, 792

Marsh, K. A., Van Cleve, J. E., Mahoney, M. J., Hayward, T. L., \& Houck, J. R. 1995, ApJ, 451, 777

Meeus, G., Waters, L. B. F. M., Bouwman, J., et al. 2001, A\&A, 365, 476

Millan-Gabet, R., Schloerb, F. P., Traub, A. W., et al. 1999, ApJ, 513, L134

Millan-Gabet, R., Schloerb, F. P., \& Traub, W. A. 2001, ApJ, 546,358

Miroshnichenko, A., Ivezić, Z̆, Vinković, D., \& Elitzur, M. 1999, ApJ, 520, L115

Nakajima, T., \& Golimowski, D. A. 1995, AJ, 109, 1181

Natta, A. 1995, RevMexAA, Serie de Conf. 1, 209
Natta, A., Grinin, V. P., \& Mannings, V. 2000, in Protostars and Planets IV, ed. V. Mannings, A. P. Boss, \& S. S. Russell (University of Arizona Press, Tucson 2000), 559 Osterloh, M., \& Beckwith, S. V. W. 1995, ApJ, 439, 288

Ridgway, S. T., Joyce, R. R., Connors, D., Pipher, J., \& Dainty, Ch. 1986, ApJ, 302, 662

Tessier, E., Bouvier, J., \& Lacombe, F. 1994, A\&A, 283, 827

Tuthill, P. G., Monnier, J. D., \& Danchi, W. C. 2001, Nature, 409, 1012

Waters, L. B. F. M., \& Waelkens, C. 1998, ARA\&A, 36, 233

Weintraub, D. A., Kastner, J. H., \& Mahesh, A. 1994, ApJ, 420, L87

Zavagno, A., Lagage, P. O., \& Cabrit, S. 1999, A\&A, 344, 499

Zinnecker, H., Perrier, C., \& Chelli, A. 1988, in High-resolution imaging by interferometry, ed. F. Merkle, ESO (Garching), part I, 505 